\title{
The Relationship between Endogenous Androgens and Body Fat Distribution in Early and Late Postmenopausal Women
}

\author{
Yuankui Cao, Shaofen Zhang*, Shien Zou, Xian Xia
}

Department of Gynecology, Obstetrics and Gynecology Hospital of Fudan University, Shanghai, China

\begin{abstract}
Objectives: To investigate the relationship between endogenous androgens and body fat distribution in early and late postmenopausal women.

Materials and Methods: We enrolled postmenopausal women consisting of an early group ( $\leq 5$ years since menopause, $n=105$ ) and a late group ( $\geq 10$ years since menopause, $n=107$ ). Each group was subdivided into normal weight (BMI $\left.<24 \mathrm{~kg} / \mathrm{m}^{2}\right)$ group, overweight and obese (BMI $\geq 24 \mathrm{~kg} / \mathrm{m}^{2}$ ) group. Fasting total testosterone (T), dehydroepiandrosteronesulfate (DHEA-S) and sex hormone-binding globulin (SHBG) levels were measured. Body fat distribution was evaluated by dual-energy X-ray absorptiometry (DEXA).

Results: Late postmenopausal women had a higher proportion of body fat than early postmenopausal women. The body fat of the overweight and obese women had a greater tendency to accumulate in the abdomen compared with the normal weight women both in early and late postmenopausal groups. The overweight and obese women had a higher free testosterone (FT) than the normal weight women in early postmenopausal women $(P<0.05)$. In late postmenopausal women, the overweight and obese women had higher DHEA-S levels than normal weight women $(P<0.05)$. No direct relationship was observed between the $T$ levels and body fat distribution both in early and late postmenopausal groups $(P>0.05)$. The FT in early postmenopausal women and the DHEA-S levels in late postmenopausal women correlated positively with the trunk/leg fat ratio (T/L) and the proportion of android fat whereas correlated negatively with the proportion of gynoid fat in the partial correlation and multiple linear regression analyses (all $P<0.05$ ).
\end{abstract}

Conclusions: Serum T levels do not correlate directly with body fat distribution, the FT in early postmenopausal women and DHEA-S levels in late postmenopausal women correlate positively with abdominal fat accumulation.

Citation: Cao Y, Zhang S, Zou S, Xia X (2013) The Relationship between Endogenous Androgens and Body Fat Distribution in Early and Late Postmenopausal Women. PLoS ONE 8(3): e58448. doi:10.1371/journal.pone.0058448

Editor: Vincent Laudet, Ecole Normale Supérieure de Lyon, France

Received July 23, 2012; Accepted February 5, 2013; Published March 4, 2013

Copyright: (C) 2013 Cao et al. This is an open-access article distributed under the terms of the Creative Commons Attribution License, which permits unrestricted use, distribution, and reproduction in any medium, provided the original author and source are credited.

Funding: This work was supported by research funding from the Shanghai Science Committee's Innovation Program (08411966000). The funders had no role in study design, data collection and analysis, decision to publish, or preparation of the manuscript.

Competing Interests: The authors have declared that no competing interests exist.

*E-mail: zhangshaofen@163.com

\section{Introduction}

Previous researchers have demonstrated that postmenopausal women tend to gain weight and that menopause is associated with a preferential increase in intra-abdominal fat $[1,2,3]$. Body fat may be redistributed and tends to accumulate more in the upper body, especially the abdomen, after menopause [4,5].It is well known that abdominal obesity significantly increases the risk of developing metabolic syndrome (MS), diabetes and coronary heart disease(CHD) [6,7], which are detrimental to the health of elderly women.

Both observational and clinical trials suggest that estrogen deficiency is associated with increased adiposity and that a low dose of estrogen therapy before 60 years could decrease abdominal fat accumulation [8,9]. Sex hormone-binding globulin (SHBG) levels decrease due to the decline of estrogens after menopause, which results in an increase of free testosterone (FT) [10]. Previous studies have suggested that elevated FT levels are associated with an accumulation of abdominal fat in premenopausal women $[11,12,13,14]$. However, few studies have been performed in postmenopausal women, and the results have not always been consistent. Furthermore, in late postmenopausal women, the majority of testosterone originates from dehydroepiandrosterone(DHEA) or dehydroepiandrosterone-sulfate(DHEA-S)in peripheral intracrine tissues [15]. Both ovaries and adrenals produce DHEA/DHEA-S. The postmenopausal ovary is hormonally active, contributes significantly to the circulating pool of androgens and this contribution appears to persist in women as long as 10 years beyond the menopause [16].However, as a result of ovarian failure, the approximately $20 \%$ contribution of the ovary to the total pool of DHEA/DHEA-S doesn't secret androgens directly [17].Thus in late postmenopausal women, the peripheral synthesis by the conversion of DHEA $\backslash$ DHEA-S originating from the adrenals becomes the main source of androgens [17,18]. DHEA sulfotransferase catalyzes the transformation of DHEA to DHEA$\mathrm{S}$ and this enzyme is mainly expressed in adrenals [19]. DHEA-S 
has a longer half-life period and is more stable in the circulation than DHEA. So DHEA-S levels could partly reflect the levels of androgens derived from adrenals in late postmenopausal women. It is unclear whether DHEA-S is associated with body fat distribution.

As ovarian ageing is a long process during natural menopause, the relationship between androgens and body fat distribution may be different in early and late postmenopausal women. In order to highlight this difference, we recruited participants in two groups according to the number of years since menopause. Therefore, the aim of our study was to evaluate the relationship between endogenous androgens and body fat distribution in early and late postmenopausal women.

\section{Materials and Methods}

\section{Participants}

This cross-sectional analysis was conducted on postmenopausal women aged 46 to 85 years who were recruited from the community around the Gynecology \& Obstetrics Hospital of Fudan University between 2009 and 2011. The study received ethics approval from the Human Ethics Committee of the Gynecology \& Obstetrics Hospital of Fudan University. All people in both groups voluntarily joined this study and signed the informed consents.

Women with a diagnosis of polycystic ovary syndrome, thyroid disease, chronic renal failure, chronic liver disease, cancer, diabetes, hypertension, coronary cardiovascular disease, or obesity due to other known diseases (e.g. cushing syndrome, hypothalamus disease) were excluded from the study. Women who were smokers; alcohol drinkers; or taking contraceptive drugs, hormone therapy medications, or any other drugs (e.g. adrenocortical hormones, yeast, chloropromazine, reserpine, isoniazide) known to interfere with body weight were also excluded. The presence of medical conditions was assessed through self-reporting.

Menopause was defined as natural menopause with amenorrhea for at least 12 months and a follicle-stimulating hormone level>40 IU/L [20,21]. Women with surgical menopause or premature ovarian failure were excluded.

A total of 212 women consisting of two groups were eligible for this study: early postmenopausal women ( $\leq 5$ years since menopause) and late postmenopausal women $(\geq 10$ years since menopause). The early postmenopause is usually defined as $\leq 5$ years since the final menstrual period (FMP) [22]. However, the definition of late postmenopause is still controversial: it was thought to be $>5$ years since the FMP in the STRAW staging system published in 2001 [22], some experts thought it should be $\geq 10$ years since the FMP, while in the STRAW staging system published in 2012 it was revised to $>6$ years since the FMP [23]. Whether the intermediate postmenopausal women (6-9 years since the FMP) are assigned to early postmenopause or late postmenopause is ambiguous and we excluded these women from this study.

Based on the guidelines defining overweight and obese Chinese adult published in 2002, a body mass index $(\mathrm{BMI}) \geq 24 \mathrm{~kg} / \mathrm{m}^{2}$ was defined as overweight and obesity [24,25]. Each menopausal group was subdivided into two groups: normal weight group $\left(\mathrm{BMI}<24 \mathrm{~kg} / \mathrm{m}^{2}\right)$, overweight and obese group $\left(\mathrm{BMI} \geq 24 \mathrm{~kg} / \mathrm{m}^{2}\right)$.

\section{Anthropometric Measurements}

Weight and height were measured in light clothing without shoes. Body height was measured using a stadiometer, and body weight was measured using a digital electronic scale.BMI was calculated as weight in kilograms divided by the square of height in meters. Waist and hip circumferences were measured using a flexible measuring tape. Waist circumference was measured midway between the xiphoid and the umbilicus during the midinspiratory phase [26]. Hip circumference was measured at the widest part of the gluteal region. The waist-to-hip ratio (WHR) was calculated as Waist circumference/Hip circumference.

A body fat composition analysis was performed using dualenergy X-ray absorptiometry (DEXA) (Discovery A, Hologic).The proportion of body fat was calculated as total fat/total mass. The proportion of android fat was defined as (trunk fat +arm fat)/(total body fat). The proportion of gynoid fat was defined as (leg fat)/ (total body fat) [27]. A high trunk/leg fat ratio (T/L), a high proportion of android fat, and a low proportion of gynoid fat were used as indications of body fat accumulation in the abdomen.

\section{Laboratory Analyses}

Participants fasted for $12 \mathrm{~h}$ and avoided heavy physical activity for $2 \mathrm{~h}$ before each examination. Fasting blood samples were collected and extracted by centrifugation at $3000 \times \mathrm{g}$ for $10 \mathrm{~min}$. Aliquots of serum were immediately stored at $-80^{\circ} \mathrm{C}$ and shipped to the hospital laboratory for long-term freezer storage until analyzed. Serum hormone concentrations were measured from stored samples. This study in which estrogen levels were not measured focused on the relationship between endogenous androgens and body fat distribution. Total testosterone(T), DHEA-S, and SHBG were determined with an immuneanaylzer using chemiluminescence kits (Beckman Coulter, Inc.). To assess the influence of free testosterone on body fat distribution, we calculated FT according to the Vermeulen method [28]. Including approximately $5 \%$ blind quality control samples in each batch of samples analyzed was performed to monitor assay variability. The quality control serum was obtained from a large pool that was aliquoted into storage vials and labeled identical to participant samples. The overall coefficients of variation were $3.93 \%$ for total T, $6.4 \%$ for DHEA-S, and $4.5 \%$ for SHBG.

\section{Statistical Analyses}

For the descriptive statistics, the means and standard deviations of the continuous variables were used to describe the study groups. Student's $t$ tests or Wilcoxon rank-sum tests were performed to compare the differences between two groups (early postmenopausal group vs. late postmenopausal group, normal weight group vs. overweight and obese group in early postmenopausal women, normal weight group vs. overweight and obese group in late postmenopausal women).Before the statistical analysis, the normal distributions and homogeneities of the variances were tested. Any parameters that did not satisfy these tests were analyzed using nonparametric rank-sum tests. The correlations between androgens and body fat distribution were evaluated using Pearson's correlation and multiple linear regression analyses. Partial correlations were adjusted for age and BMI. The model ${ }^{1}$ used in multiple linear regression analysis included T, DHEA-S, SHBG, BMI and age. FT was added to model ${ }^{1 \mathrm{a}}$. DHEA-S, FT, BMI and age were included in model ${ }^{2}$. SHBG was added to model ${ }^{2 a}$.All statistical analyses were performed using SPSS 17.0 software. The statistical significance level was set at $P<0.05$.

\section{Results}

The patient characteristics (mean $\pm \mathrm{SD}$ ) for each study group are shown in Table 1. Late postmenopausal women had a higher proportion of body fat compared with early postmenopausal women $(34.86 \pm 4.29 \%$ vs. $33.05 \pm 3.50 \% ; P<0.01)$. No significant differences were observed in the body fat distribution, BMI, or 
Table 1. Comparisons of participants' characteristics between two groups.

\begin{tabular}{|c|c|c|c|}
\hline & \multirow{2}{*}{$\begin{array}{l}\text { early postmenopausal women } \\
\mathrm{N}=105\end{array}$} & \multirow{2}{*}{$\begin{array}{l}\text { late postmenopausal women } \\
\mathrm{N}=107\end{array}$} & \multirow[t]{2}{*}{$p$} \\
\hline & & & \\
\hline Ages(years) & $54.58 \pm 2.98$ & $69.41 \pm 7.28$ & $<0.01$ \\
\hline Years since menopause & $3.50 \pm 1.48$ & $19.35 \pm 7.50$ & $<0.01$ \\
\hline Weight(kg) & $60.47 \pm 7.06$ & $58.58 \pm 10.07$ & NS \\
\hline Height(m) & $1.59 \pm 0.05$ & $1.58 \pm 0.05$ & NS \\
\hline $\operatorname{BMI}\left(\mathrm{kg} / \mathrm{m}^{2}\right)$ & $24.06 \pm 2.72$ & $23.52 \pm 3.28$ & NS \\
\hline Waist circumferences(cm) & $78.78 \pm 7.71$ & $77.63 \pm 10.21$ & NS \\
\hline Hip circumferences(cm) & $92.96 \pm 5.36$ & $92.70 \pm 7.88$ & NS \\
\hline WHR & $0.85 \pm 0.05$ & $0.84 \pm 0.06$ & NS \\
\hline $\mathrm{T}(\mathrm{ng} / \mathrm{mL})$ & $0.26 \pm 0.15$ & $0.25 \pm 0.15$ & NS \\
\hline DHEA-S( $\mu \mathrm{g} / \mathrm{dL})$ & $112.06 \pm 53.19$ & $92.88 \pm 49.28$ & $<0.05$ \\
\hline SHBG(nmol/L) & $61.79 \pm 38.12$ & $64.05 \pm 27.47$ & NS \\
\hline $\mathrm{FT}(\mathrm{pmol} / \mathrm{L})$ & $11.23 \pm 9.93$ & $10.07 \pm 7.93$ & NS \\
\hline \multicolumn{4}{|l|}{ Body fat distribution } \\
\hline Total mass(kg) & $59.14 \pm 7.15$ & $57.57 \pm 10.21$ & NS \\
\hline Trunk fat(kg) & $10.21 \pm 2.46$ & $10.58 \pm 3.23$ & NS \\
\hline Left arm fat $(\mathrm{kg})$ & $1.23 \pm 0.27$ & $1.36 \pm 0.50$ & NS \\
\hline Right arm fat(kg) & $1.28 \pm 0.30$ & $1.42 \pm 0.56$ & NS \\
\hline Left leg fat(kg) & $3.03 \pm 0.70$ & $3.06 \pm 0.99$ & NS \\
\hline Right leg fat(kg) & $3.12 \pm 0.74$ & $3.11 \pm 1.01$ & NS \\
\hline Total fat(kg) & $19.68 \pm 3.88$ & $20.37 \pm 5.96$ & NS \\
\hline Proportion of body fat(\%) & $33.05 \pm 3.50$ & $34.86 \pm 4.29$ & $<0.01$ \\
\hline$T / L$ & $1.70 \pm 0.40$ & $1.69 \pm 0.17$ & NS \\
\hline Proportion of android fat & $0.64 \pm 0.05$ & $0.65 \pm 0.04$ & NS \\
\hline Proportion of gynoid fat & $0.31 \pm 0.05$ & $0.30 \pm 0.04$ & NS \\
\hline
\end{tabular}

Results are expressed as mean $\pm S D$. NS, no significant difference; $P<0.05$, significant difference.

doi:10.1371/journal.pone.0058448.t001

WHR between the groups (all $P>0.05$ ). Early postmenopausal women had higher DHEA-S (1 12.06 \pm 53.19 vs. $92.88 \pm 49.28 \mu \mathrm{g} /$ $\mathrm{dL} ; P<0.05)$ levels than late postmenopausal women. Early and late postmenopausal women had similar T,FT and SHBG levels.

After adjustment for BMI, we found that overweight and obese women had a higher $\mathrm{T} / \mathrm{L}$, a higher proportion of android fat, and a lower proportion of gynoid fat than normal weight women both in the early and late postmenopausal groups (all $P<0.05$, Table 2 ). In the early postmenopausal group, overweight and obese women had higher DHEA-S and T levels than normal weight women, but these differences were not significant. Overweight and obese women had a higher FT $(13.85 \pm 11.47$ vs. $8.49 \pm 7.15 \mathrm{pmol} / \mathrm{L}$; $P<0.05)$ and lower SHBG levels $(52.30 \pm 37.32$ vs. $71.29 \pm 36.93 \mathrm{nmol} / \mathrm{L} ; P<0.05)$ than normal weight women in the early postmenopausal group. In the late postmenopausal group, overweight and obese women had higher DHEA-S $(106.10 \pm 52.64$ vs. $79.65 \pm 42.78 \mu \mathrm{g} / \mathrm{dL} ; P<0.05)$ levels, higher $\mathrm{T}$ levels and FT values (not significant), lower SHBG levels (not significant) than normal weight women.

The Pearson's correlation analyses indicated that there were no direct relationships between the $\mathrm{T}$ levels and body fat distribution both in early postmenopausal women and late postmenopausal women (all $P>0.05$, Table 3 ).

In the Pearson's correlation analysis, SHBG levels correlated negatively with the T/L $(\mathrm{r}=-0.221, P=0.034$; Table 3$)$ and the proportion of android fat $(\mathrm{r}=-0.279, \quad P=0.007$; Table 3), whereas they correlated positively with the proportion of gynoid fat $(\mathrm{r}=0.270, P=0.009$; Table 3$)$ in early postmenopausal women after adjustment for age and BMI. The FT correlated positively with the T/L $(\mathrm{r}=0.339, P=0.001$; Table 3$)$ and the proportion of android fat $(\mathrm{r}=0.227, P=0.03$; Table 3$)$, while FT correlated negatively with the proportion of gynoid fat $(\mathrm{r}=-0.231$, $P=0.027$; Table 3$)$ in early postmenopausal women. In the late postmenopausal group, after adjustment for age and BMI, the DHEA-S levels correlated positively with $\mathrm{T} / \mathrm{L} \quad(\mathrm{r}=0.297$, $P=0.023$; Table 3$)$ and the proportion of android fat $(\mathrm{r}=0.282$, $P=0.032$; Table 3 ), whereas they correlated negatively with the proportion of gynoid fat $(\mathrm{r}=-0.277, P=0.035$; Table 3$)$.

The results from multiple linear regression analyses are shown in Table 4. In early postmenopausal women SHBG levels correlated negatively with the $\mathrm{T} / \mathrm{L} \quad\left(\operatorname{model}^{1}, \beta=-0.235\right.$, $P=0.023$; Table 4) and the proportion of android fat (mod$\mathrm{el}^{1}, \beta=-0.267, P=0.007$; Table 4), whereas they correlated positively with the proportion of gynoid fat $\left(\operatorname{model}^{1}, \beta=0.280\right.$, $P=0.006$; Table 4).A higher FT in early postmenopausal women was significantly associated with a higher $\mathrm{T} / \mathrm{L}$ (mode$1^{1 \mathrm{a}}, \beta=0.367, P=0.003 ; \mathrm{model}^{2}, \beta=0.302, P=0.002 ; \mathrm{model}^{2 \mathrm{a}},-$

$\beta=0.279, P=0.005$; Table 4$)$, higher proportion of android fat $(\mathrm{mo}-$ $\mathrm{del}^{\mathrm{la}}, \quad \quad \beta=0.283, \quad P=0.047 ; \mathrm{model}^{2}, \quad \beta=0.225$, $P=0.030 ;$ model $^{2 \mathrm{a}}, \beta=0.208, P=0.043$; Table 4 ) and lower proportion of gynoid fat $\left(\right.$ model $^{1 \mathrm{a}}, \beta=-0.296, P=0.047 ; \operatorname{model}^{2}, \beta=-0.244, P=0.023 ;-$ 
Table 2. Body fat distribution and androgens after adjustment for BMI.

\begin{tabular}{|c|c|c|c|c|}
\hline & \multicolumn{2}{|c|}{ early postmenopausal women } & \multicolumn{2}{|c|}{ late postmenopausal women } \\
\hline & BMI $<24$ & $B M I \geq 24$ & $B M I \geq 24$ & $\mathrm{BMI} \geq 24$ \\
\hline & $n=53$ & $\mathbf{n}=\mathbf{5 2}$ & $n=53$ & $n=54$ \\
\hline$T / L$ & $1.60 \pm 0.35$ & $1.81 \pm 0.42^{*}$ & $1.63 \pm 0.11$ & $1.74 \pm 0.20^{* *}$ \\
\hline Proportion of android fat & $0.63 \pm 0.05$ & $0.66 \pm 0.04^{*}$ & $0.63 \pm 0.04$ & $0.67 \pm 0.04^{* *}$ \\
\hline Proportion of gynoid fat & $0.33 \pm 0.05$ & $0.30 \pm 0.04^{*}$ & $0.32 \pm 0.04$ & $0.29 \pm 0.05^{* *}$ \\
\hline DHEA-S $(\mu \mathrm{g} / \mathrm{dL})$ & $101.50 \pm 50.87$ & $122.62 \pm 53.94$ & $79.65 \pm 42.78$ & $106.10 \pm 52.64^{* *}$ \\
\hline $\mathrm{T}(\mathrm{ng} / \mathrm{mL})$ & $0.22 \pm 0.13$ & $0.29 \pm 0.15$ & $0.22 \pm 0.13$ & $0.28 \pm 0.16$ \\
\hline SHBG (nmol/L) & $71.29 \pm 36.93$ & $52.30 \pm 37.32^{*}$ & $70.93 \pm 27.03$ & $57.16 \pm 26.70$ \\
\hline FT(pmol/L) & $8.49 \pm 7.15$ & $13.85 \pm 11.47^{*}$ & $7.08 \pm 5.65$ & $13.16 \pm 8.82$ \\
\hline
\end{tabular}

${ }^{*} p<0.05$, overweight women vs. normal weight women in the early postmenopausal group.

${ }^{* *} p<0.05$, overweight women vs. normal weight women in the late postmenopausal group.

doi:10.1371/journal.pone.0058448.t002

model $^{2 \mathrm{a}}, \beta=-0.223, P=0.030 ;$ Table 4$)$. Higher DHEA-S levels in late postmenopausal women were significantly associated with higher T/L (model ${ }^{1,1 a, 2,2 a}, \beta=0.288, P=0.026$; Table 4), higher proportion of android fat (model ${ }^{1,1 \mathrm{a}, 2,2 \mathrm{a}}, \beta=0.251, P=0.047$; Table 4) and lower proportion of gynoid fat (mode$1^{1,1 \mathrm{a}, 2,2 \mathrm{a}}, \beta=-0.302, P=0.019$; Table 4).

\section{Discussion}

Body fat is harmful to health depending on its distribution. There is a marked sex difference in the body fat distribution between men and women. Men tend to accumulate fat in the abdomen (android fat distribution), whereas women tend to accumulate fat in the gluteal-femoral region (gynoid fat distribution) [29].An increased android fat distribution and a decreased gynoid fat distribution which are thought to represent abdominal fat accumulation are risk factors for CHD and MS in women [30].

Natural menopause is associated with increased abdominal fat that exceeds changes normally attributed to the aging process, primarily in the perimenopausal year $[8,31,32]$. Evidences from estrogen therapy have demonstrated that the declined levels of estrogens after menopause are associated with abdominal fat accumulation [9].However, to date the relationship between endogenous androgens and body fat distribution is unclear.

Ovarian production of testosterone remains relatively stable in early postmenopause, in fact, the relative contribution of the ovarian testosterone to total testosterone increases [33]. SHBG is the main transport protein for testosterone to target tissues and modulates their biological activity. In this study, overweight and obese women of early postmenopausal group had lower SHBG levels than women with normal weight, which suggested that SHBG levels correlated negatively with abdominal fat accumulation in early postmenopausal women. Results from partial correlation and multiple linear regression analyses (in model $^{1}$ )confirmed this. Our results were in agreement with previous reports that low SHBG levels correlated with abdominal obesity in postmenopausal women [26,34].

In our study, the FT correlated positively with abdominal fat accumulation in early postmenopausal women in model ${ }^{2}$. We further tested in model $^{1 \mathrm{a}, 2 \mathrm{a}}$ including FT and SHBG the relationship between androgens and body fat distribution. In-

Table 3. The relationship between androgens and body fat distribution in early and late postmenopausal groups after adjustment for age and BMI.

\begin{tabular}{|c|c|c|c|c|c|c|}
\hline & \multicolumn{2}{|l|}{$T / L$} & \multicolumn{2}{|c|}{ Proportion of android fat } & \multicolumn{2}{|c|}{ Proportion of gynoid fat } \\
\hline & r-value & p-value & r-value & p-value & r-value & p-value \\
\hline \multicolumn{7}{|c|}{ early postmenopausal women } \\
\hline $\mathrm{T}$ & 0.076 & 0.474 & -0.026 & 0.805 & 0.017 & 0.874 \\
\hline SHBG & -0.221 & 0.034 & -0.279 & 0.007 & 0.270 & 0.009 \\
\hline DHEA-S & 0.003 & 0.975 & 0.034 & 0.749 & -0.017 & 0.869 \\
\hline FT & 0.339 & 0.001 & 0.227 & 0.030 & -0.231 & 0.027 \\
\hline \multicolumn{7}{|c|}{ late postmenopausal women } \\
\hline $\mathrm{T}$ & -0.055 & 0.680 & 0.119 & 0.373 & -0.109 & 0.413 \\
\hline SHBG & 0.006 & 0.964 & -0.064 & 0.631 & 0.077 & 0.564 \\
\hline DHEA-S & 0.297 & 0.023 & 0.282 & 0.032 & -0.277 & 0.035 \\
\hline $\mathrm{FT}$ & -0.050 & 0.710 & 0.128 & 0.340 & -0.122 & 0.362 \\
\hline
\end{tabular}

Results are reported as Pearson's rank value correlation coefficient ( $r$-value).

Correlation is significant at the 0.05 level $(P<0.05)$.

doi:10.1371/journal.pone.0058448.t003 
Table 4. Associations of androgens with body fat distribution by multiple linear regressions analysis in early and late postmenopausal groups.

\begin{tabular}{|c|c|c|c|c|c|c|}
\hline & \multicolumn{2}{|l|}{$\mathbf{T} / \mathbf{L}$} & \multicolumn{2}{|c|}{ Proportion of android fat } & \multicolumn{2}{|c|}{ Proportion of gynoid fat } \\
\hline & $\beta$-value & p-value & $\beta$-value & p-value & $\beta$-value & p-value \\
\hline \multicolumn{7}{|c|}{ early postmenopausal women } \\
\hline Model ${ }^{1}$ SHBG & -0.235 & 0.023 & -0.267 & 0.007 & 0.280 & 0.006 \\
\hline Model ${ }^{1 a} \mathrm{FT}$ & 0.367 & 0.003 & 0.283 & 0.047 & -0.296 & 0.047 \\
\hline Model $^{2}$ FT & 0.302 & 0.002 & 0.225 & 0.030 & -0.244 & 0.023 \\
\hline Model ${ }^{2 a} \mathrm{FT}$ & 0.279 & 0.005 & 0.208 & 0.043 & -0.223 & 0.030 \\
\hline \multicolumn{7}{|c|}{ late postmenopausal women } \\
\hline Model $^{1,1 a, 2,2 a}$ DHEA-S & 0.288 & 0.026 & 0.251 & 0.047 & -0.302 & 0.019 \\
\hline
\end{tabular}

terestingly, the FT was positively and significantly associated with abdominal fat accumulation though the strength of the association was a little different in different models, whereas the relationship between SHBG and body fat distribution was no longer significant. These results suggested that SHBG was not an independent factor associated with abdominal fat accumulation and the FT was independent from SHBG and $\mathrm{T}$ to predict abdominal fat accumulation.

In late postmenopause, the ovarian production of androstenedione declines, the DHEA-S from adrenal gland becomes the primary source of this precursor [35]. Serum DHEA-S levels decrease markedly during aging [15]. We also found that late postmenopausal women had lower DHEA-S levels than early postmenopausal women. Some researchers have reported that DHEA therapy in postmenopausal women could improve syndromes that result from androgen deficiency [36,37]. DHEA is even sold over the counter in the USA as a nutritional supplement. However, due to the lack of definitive evidence for biological mechanisms and safety, DHEA therapy for postmenopausal is not recommended [38,39].

The reduction of DHEA-S during aging results in a dramatic reduction in the formation of androgens in peripheral tissues, which is thought to be associated with obesity in men [40]. Conversely,this study showed that overweight and obese women had higher DHEA-S levels than normal weight women in the late postmenopausal group. The partial correlation and multiple linear regression analyses (in model ${ }^{1,1 a, 2,2 a}$ ) further showed a positive relationship between DHEA-S and abdominal fat accumulation. Our findings were consistent with the findings that endogenous DHEA-S levels were positively associated with insulin resistance which is one of the complications of abdominal obesity [41]. This study suggested that DHEA therapy should be administered cautiously in late postmenopausal women, especially in women with overweight and obesity. Due to relatively higher endogenous DHEA-S levels in late postmenopausal women with overweight and obesity, exogenous DHEA therapy could cause more DHEA accumulation in peripheral tissues. As DHEA-S converts more readily to androgens than to estrogens [42], higher DHEA-S levels result in an increase in testosterone production in peripheral tissues, which may cause side effects(e.g. insulin resistance, acne, hypertrichiasis) due to higher testosterone.

We did not find a direct relationship between serum $\mathrm{T}$ levels and body fat distribution. This may be because the majority of circulating testosterone bounds to SHBG, and only FT expresses a biological effect. Serum T levels, therefore, do not fully represent the levels of bioavailable testosterone. Additionally, testosterone is mainly locally synthesized in the peripheral tissues from DHEA-S in late postmenopausal women without relying on transport by SHBG. After inducing their biological effects, most testosterone is inactivated immediately in the same cells $[15,43]$. The serum levels of testosterone cannot indicate the levels of this bioavailable testosterone in peripheral tissues.

The proportion of body fat increases with aging and postmenopausal status is associated with abdominal fat accumulation $[2,44]$. We also found that late postmenopausal women had a $1.81 \%$ higher proportion of body fat than early postmenopausal women due to aging. Body fat tended to accumulate in the abdomen in overweight and obese postmenopausal women. Previous studies indicated that the onset time of this tendency was early postmenopause in which the ovaries were hormonally active for secreting testosterone [2,8]. Adipose tissue is known to express several steroidogenic and steroid-inactivating enzymes which covert DHEA-S to testosterone [45]. With ageing more testosterone is produced from DHEA-S and inactivated in adipose tissues rather than transported by SHBG from ovaries. Maybe the different sources of androgens accounted for the different correlations in early and late postmenopausal women. However, whether menopause-related changes in endogenous androgens account for body fat distribution or whether body fat distribution drives changes in endogenous androgens required further study.

In conclusion, our study suggested that the FT in early postmenopausal women and DHEA-S levels in late postmenopausal women correlated positively with abdominal fat accumulation.

\section{Acknowledgments}

This work was supported by the radiology department and clinical laboratory of Gynecology \& Obstetrics Hospital of Fudan University. We thank all the people participating in this study.

\section{Author Contributions}

Conceived and designed the experiments: YC S. Zhang S. Zou XX. Performed the experiments: YC. Analyzed the data: YC S. Zhang S. Zou XX. Wrote the paper: YC S. Zhang. 


\section{References}

1. Folsom AR, Kushi LH, Anderson KE, Mink PJ, Olson JE, et al. (2000) Associations of general and abdominal obesity with multiple health outcomes in older women: the Iowa Women's Health Study. Arch Intern Med 160: 2117 2128.

2. Toth MJ, Tchernof A, Sites CK, Poehlman ET (2000) Menopause-related changes in body fat distribution. Ann N Y Acad Sci 904: 502-506.

3. Llaneza P, Inarrea J, Gonzalez C, Alonso A, Arnott I, et al. (2007) Differences in health related quality of life in a sample of Spanish menopausal women with and without obesity. Maturitas 58: 387-394.

4. Panotopoulos G, Ruiz JC, Raison J, Guy-Grand B, Basdevant A (1996) Menopause, fat and lean distribution in obese women. Maturitas 25: 11-19.

5. Tremollieres FA, Pouilles JM, Ribot CA (1996) Relative influence of age and menopause on total and regional body composition changes in postmenopausal women. Am J Obstet Gynecol 175: 1594-1600.

6. Janssen I, Heymsfield SB, Allison DB, Kotler DP, Ross R (2002) Body mass index and waist circumference independently contribute to the prediction of nonabdominal, abdominal subcutaneous, and visceral fat. Am J Clin Nutr 75: 683-688.

7. He Q, Horlick M, Fedun B, Wang J, Pierson RN Jr, et al. (2002) Trunk fat and blood pressure in children through puberty. Circulation 105: 1093-1098.

8. Tchernof A, Poehlman ET (1998) Effects of the menopause transition on body fatness and body fat distribution. Obes Res 6: 246-254

9. Salpeter SR, Walsh JM, Ormiston TM, Greyber E, Buckley NS, et al. Metaanalysis: effect of hormone-replacement therapy on components of the metabolic syndrome in postmenopausal women. Diabetes Obes Metab 2006;8: 538-54.

10. Burger HG, Dudley EC, Cui J, Dennerstein L, Hopper JL (2000) A prospective longitudinal study of serum testosterone, dehydroepiandrosterone sulfate, and sex hormone-binding globulin levels through the menopause transition. J Clin Endocrinol Metab 85: 2832-2838.

11. Ivandic A, Prpic-Krizevac I, Bozic D, Barbir A, Peljhan V, et al. (2002) Insulin resistance and androgens in healthy women with different body fat distributions. Wien Klin Wochenschr 114: 321-326.

12. De Pergola G, Triggiani V, Giorgino F, Cospite MR, Garruti G, et al. (1994) The free testosterone to dehydroepiandrosterone sulphate molar ratio as a marker of visceral fat accumulation in premenopausal obese women. Int $\mathrm{J}$ Obes Relat Metab Disord 18: 659-664.

13. Tchernof A, Toth MJ, Poehlman ET (1999) Sex hormone-binding globulin levels in middle-aged premenopausal women. Associations with visceral obesity and metabolic profile. Diabetes Care 22: 1875-1881.

14. Hajamor S, Despres JP, Couillard C, Lemieux S, Tremblay A, et al. (2003) Relationship between sex hormone-binding globulin levels and features of the metabolic syndrome. Metabolism 52: 724-730.

15. Labrie F, Belanger A, Luu-The V, Labrie C, Simard J, et al. (1998) DHEA and the intracrine formation of androgens and estrogens in peripheral target tissues: its role during aging. Steroids 63: 322-328.

16. Fogle RH, Stanczyk FZ, Zhang X, Paulson RJ (2007) Ovarian androgen production in postmenopausal women. J Clin Endocrinol Metab 92: 3040-3043.

17. Labrie F, Martel C, Balser J (2011) Wide distribution of the serum dehydroepiandrosterone and sex steroid levels in postmenopausal women: role of the ovary. Menopause 18: 30-43.

18. Labrie F, Luu-The V, Labrie G, Simard J (2001) DHEA and its transformation into androgens and estrogens in peripheral target tissues: intracrinology. Front Neuroendocrinol 22: 185-212.

19. Luu-The V, Dufort I, Paquet N, Reimnitz G, Labrie F (1995) Structural characterization and expression of the human dehydroepiandrosterone sulfotransferase gene. DNA Cell Biol 14: 511-518.

20. Kahwati LC, Haigler L, Rideout S, Markova T (2005) What is the best way to diagnose menopause. J Fam Pract 54: 1000-1002.

21. Shelling AN (2010) Premature ovarian failure. Reproduction 140: 633-641.

22. Soules MR, Sherman S, Parrott E, Rebar R, Santoro N, et al. (2001) Executive summary: Stages of Reproductive Aging Workshop (STRAW). Climacteric 4: $267-272$.
23. Harlow SD, Gass M, Hall JE, Lobo R, Maki P, et al. (2012) Executive summary of the Stages of Reproductive Aging Workshop +10: addressing the unfinished agenda of staging reproductive aging. Climacteric 15: 105-114.

24. Zhou B (2002) Predictive values of body mass index and waist circumference to risk factors of related diseases in Chinese adult population]. Zhonghua Liu Xing Bing Xue Za Zhi 23: 5-10.

25. Bei-Fan $Z$ (2002) Predictive values of body mass index and waist circumference for risk factors of certain related diseases in Chinese adults: study on optimal cutoff points of body mass index and waist circumference in Chinese adults. Asia Pac J Clin Nutr 11 Suppl 8: S685-693.

26. Akin F, Bastemir M, Alkis E, Kaptanoglu B (2009) SHBG levels correlate with insulin resistance in postmenopausal women. Eur J Intern Med 20: 162-167.

27. Kirchengast S, Gruber D, Sator M, Hartmann B, Knogler W, et al. (1997) Menopause-associated differences in female fat patterning estimated by dualenergy X-ray absorptiometry. Ann Hum Biol 24: 45-54.

28. Vermeulen A, Verdonck L, Kaufman JM (1999) A critical evaluation of simple methods for the estimation of free testosterone in serum. J Clin Endocrinol Metab 84: 3666-3672.

29. Blouin K, Boivin A, Tchernof A (2008) Androgens and body fat distribution. J Steroid Biochem Mol Biol 108: 272-280.

30. Kirchengast S, Huber J (2004) Body composition characteristics and fat distribution patterns in young infertile women. Fertil Steril 81: 539-544.

31. Poehlman ET, Toth MJ, Gardner AW (1995) Changes in energy balance and body composition at menopause: a controlled longitudinal study. Ann Intern Med 123: 673-675.

32. Svendsen OL, Hassager C, Christiansen C (1995) Age- and menopauseassociated variations in body composition and fat distribution in healthy women as measured by dual-energy X-ray absorptiometry. Metabolism 44: 369-373.

33. Vermeulen A (1976) The hormonal activity of the postmenopausal ovary. J Clin Endocrinol Metab 42: 247-253.

34. Weinberg ME, Manson JE, Buring JE, Cook NR, Seely EW, et al. (2006) Low sex hormone-binding globulin is associated with the metabolic syndrome in postmenopausal women. Metabolism 55: 1473-1480.

35. Cobin RH, Futterweit W, Ginzburg SB, Goodman NF, Kleerekoper M, et al. (2006) American Association of Clinical Endocrinologists medical guidelines for clinical practice for the diagnosis and treatment of menopause. Endocr Pract 12: 315-337.

36. Schmidt PJ, Daly RC, Bloch M, Smith MJ, Danaceau MA, et al. (2005) Dehydroepiandrosterone monotherapy in midlife-onset major and minor depression. Arch Gen Psychiatry 62: 154-162.

37. Hackbert L, Heiman JR (2002) Acute dehydroepiandrosterone (DHEA) effects on sexual arousal in postmenopausal women. J Womens Health Gend Based Med 11: 155-162.

38. Genazzani AR, Pluchino N (2010) DHEA therapy in postmenopausal women: the need to move forward beyond the lack of evidence. Climacteric 13: 314-316.

39. Davis SR, Panjari M, Stanczyk FZ (2011) Clinical review: DHEA replacement for postmenopausal women. J Clin Endocrinol Metab 96: 1642-1653.

40. Nestler JE, Barlascini CO, Clore JN, Blackard WG (1988) Dehydroepiandrosterone reduces serum low density lipoprotein levels and body fat but does not alter insulin sensitivity in normal men. J Clin Endocrinol Metab 66: 57-61.

41. Golden SH, Dobs AS, Vaidya D, Szklo M, Gapstur S, et al. (2007) Endogenous sex hormones and glucose tolerance status in postmenopausal women. J Clin Endocrinol Metab 92: 1289-1295.

42. Labrie F, Belanger A, Belanger P, Berube R, Martel C, et al. (2007) Metabolism of DHEA in postmenopausal women following percutaneous administration. J Steroid Biochem Mol Biol 103: 178-188.

43. Labrie F (2003) Extragonadal synthesis of sex steroids: intracrinology. Ann Endocrinol (Paris) 64: 95-107.

44. Douchi T, Yamamoto S, Yoshimitsu N, Andoh T, Matsuo T, et al. (2002) Relative contribution of aging and menopause to changes in lean and fat mass in segmental regions. Maturitas 42: 301-306.

45. Belanger C, Luu-The V, Dupont P, Tchernof A (2002) Adipose tissue intracrinology: potential importance of local androgen/estrogen metabolism in the regulation of adiposity. Horm Metab Res 34: 737-745. 